\title{
Response to the Letter to the Editor for "Pharmacotherapy for the Treatment of Weight Recidivism or Weight-Loss Plateau Post-Bariatric Surgery"
}

\author{
Jennifer S. Schwartz ${ }^{1}$ Andrew Suzo ${ }^{1}$ Sabrena F. Noria ${ }^{1}$
}

Published online: 29 March 2016

(C) Springer Science+Business Media New York 2016

To the Editor:

We would like to thank the authors of the letter to the editor for their thoughtful response to our manuscript in which we advocate the use of pharmacotherapy as an adjunct for treatment of weight recidivism or weight-loss plateau following bariatric surgery. We concur with the authors that, given the growing interest in this therapeutic option, there is a need for larger, prospective trials to evaluate the use of pharmacotherapy in this patient population. Additionally, we agree that these investigations should not be restricted to the use of weight-lossspecific medications, but should also include the use of GLP-1 agonists given the changes in gut hormone levels following surgery. Our study, along with those cited by the author of the letter, indicates the growing interest in the use of pharmacotherapy in this complicated patient

population and helps to build a foundation for larger, prospective trials to evaluate the validity and effectiveness of this therapy in those that have undergone any type of bariatric procedure. We look forward to the continued investigation of pharmacotherapy as a viable treatment option for patients who have had weight recidivism or weight-loss plateau following bariatric surgery.

Compliance with Ethical Standards

Conflict of Interest The authors declare that they have no conflict of interest.

Ethical Approval Ethical approval and informed consent are not applicable.

Jennifer S. Schwartz

jennifer.schwartz@osumc.edu

Andrew Suzo

Andrew.suzo@osumc.edu

Sabrena F. Noria

Sabrena.noria@osumc.edu

1 Center for Minimally Invasive Surgery, The Ohio State University Wexner Medical Center, 410 W. 10th Ave, Room 558,

Columbus, OH 43210, USA 